\title{
Ambiente de aprendizaje para la enseñanza de las matemáticas ante el COVID-19
}

\author{
Learning environment for teaching mathematics in the face of COVID-19
}

William Oswaldo Flores López ${ }^{1}$

\section{Resumen}

Esta investigación ha identificado la percepción del profesorado respecto a los ambientes educativos en la enseñanza de las matemáticas ante el COVID-19. Se trata de una investigación con enfoque cuantitativo de naturaleza descriptiva. Los datos obtenidos son el resultado del suministro de un cuestionario online a 83 profesores de Educación Secundaria de Nicaragua. Se realizó un análisis descriptivo de los datos y los resultados muestran que, la percepción del profesorado genera un valor positivo en aspectos: tecnológicos, cognitivos, aprendizaje y enseñanza, diversidad en la población, contenidos disciplinarios, campo del conocimiento y diversidad como orientación. Se concluye que, el diseño de ambientes de aprendizajes donde confluye la interculturalidad y la tecnología mejoraría la educación de las matemáticas para poblaciones en contexto de diversidad.

Palabras Clave: Ambientes; Enseñanza; Aprendizaje; Matemáticas; Diversidad.

\section{Abstract}

This research has identified the teachers' perception related to the learning environments for the teaching of mathematics in the face of COVID - 19. It is about a research with a quantitative approach with a descriptive nature, the obtained information is the result of the application of an online questionnaire addressed to 83 High School teachers from Nicaragua. A descriptive analysis from the data was made and the results show that the perception of teachers generates a positive value in characteristics such as: technological, cognitive, learning and teaching processes; population diversity, subject contents, knowledge fields and diversity as a transversal axis. As a conclusion, the learning environment design where interculturality and technology converges, there is an improvement on the teaching and learning mathematics for populations in contexts of diversity.

Keywords: Environments; Teaching; Learning; Mathematics; Diversity.

1 Doctor en Educación, Profesor Investigador de la Universidad de las Regiones Autónomas de la Costa Caribe Nicaragüense. Email: william.flores@uraccan.edu.ni ORCID: https://orcid.org/oooo-ooo2-1016-1620

Recibido: 08/01/2021 - Aprobado: 05/02/2021 


\section{Introducción}

El contexto del COVID-19 ha ralentizado los procesos de aprendizajes en las Instituciones de Educación Superior de Nicaragua; entonces, surgen situaciones vivenciales y contextuales históricamente como: (i) Los desafíos de formación y actualización pedagógica y tecnológica de docentes para que incorporen las tecnologías del aprendizaje en un ambiente propiciado por las TIC (Flores, 2018); (ii) La carencia de recursos didácticos y tecnológicos, re-organizados para los procesos de aprendizaje de las matemáticas (Flores, 2016); y (iii) La disparidad de los logros de aprendizaje y la desigualdades de acceso a la educación que evidencia una brecha digital (IESALC, 2008).

Desde esta perspectiva, se generalizan las dificultades de aprendizajes en las matemáticas por falta de proceso de evaluación con el uso de herramientas colaborativas mediados por las tecnologías; además, la poca experiencia en la docencia virtual del profesorado produce un vacío de mentoría y acompañamientos, así mismo, la mediación didáctica y tecnológica no es factible en los procesos de aprendizaje por la saturación de actividades. En este sentido, se trata de identificar la percepción del profesorado sobre ambientes de aprendizajes para la enseñanza de las matemáticas ante el COVID-19, mediante la aplicación de un cuestionario online con ayuda de Google Formulario.

\section{Revisión de literatura}

El desarrollo del aprendizaje matemático, demanda del profesorado conocimientos, saberes, capacidades, valores, principios, actitudes humanistas, sentido del emprendimiento e innovación para construir ambientes para la enseñanza de las matemáticas. Ambientes que no pueden limitarse a distribuir contenidos, sino en el supuesto de proponer un conjunto de actividades y experiencias en las que participan los estudiantes, teniendo como referencia la diversidad y la incorporación de la tecnología. Parte de este interés es el cuerpo de investigación que ha comenzado a construirse en torno a ambientes interculturales y pluritecnológicos (Cano y Lledo, 1995; Flores y Auzmendi, 2015).

Se trata de ambientes que constituyen un recurso útil para favorecer procesos de aprendizajes de las matemáticas en contextos multiculturales, es decir, son diseños didácticos al servicio de la educación matemática en y para la diversidad. Por tales razones, un hacer natural del profesorado de matemática es seleccionar, organizar y planificar las experiencias de aprendizajes necesarias para que el estudiantado aprenda en contexto diversos. La gestión didáctica de los ambientes de aprendizajes, las interacciones entre los estudiantes y las perspectivas del aprendizaje y sus variables asociadas (motivación, actitudes) aparecen en un eje central para propiciar líneas de investigación. Junto a esta perspectiva centrada en el aprendizaje destacan también las cuestiones relacionadas con el coste, la accesibilidad universal a la enseñanza superior 
(Karsenti, 2013), el problema de las tasas de deserción de los estudiantes (Fiegehen, 2005), además de la problemática de su integración en los estudios formales.

El desarrollo de ambientes educativos en entornos escolares exige considerar el currículo desde una perspectiva ambiental de la educación que necesariamente incorpora aspectos ecológicos, psicológicos y comunicativos, los cuales se articulan con propósitos didácticos para constituir una perspectiva bioecológica, tanto en la educación en general como en la didáctica de las matemáticas en particular (Calderón, León, e INCE, 2010). En el ambiente de aprendizaje ingresan, se instauran y se desarrollan formas de trabajo, de relaciones socioculturales y discursivas que están relacionadas con el estudio; también participan en la reflexión y la solución de problemas alrededor de temas escolares. Las cuales consideran prioritariamente condiciones de edad, género, desarrollo intelectual y social, así como condiciones físicas, fisiológicas y sensoriales, con el fin de adecuar todos los aspectos intervinientes para facilitar el trabajo escolar en ese ambiente (León et al., 2014). Los ambientes de aprendizaje proporcionan condiciones para que todas las personas del grupo tengan acceso al conocimiento y actividades propuestas, así como el contacto con los materiales para abarcar un amplio abanico de aprendizajes cognitivos, afectivos y sociales (Calderón y León, 2010).

La educación matemática en contextos multiculturales y con pertinencia social tiene como propósito desarrollar habilidades para la convivencia social entre diversos y eliminar las brechas educativas entre diferentes tipos de poblaciones (León et al. 2014). Desde esta perspectiva, la educación matemática está orientada al fortalecimiento de competencias científico-técnicas para el avance de la educación en la autonomía regional, el desarrollo con identidad apuntando hacia al buen vivir, vivir bien y el vivir con dignidad en una ciudadanía intercultural. En el aprendizaje de las matemáticas, las tareas, actividades o situaciones problema que articulan los ambientes de aprendizaje, tienen como objetivo posibilitar que los estudiantes re- signifiquen su conocimiento matemático y didáctico, ampliándolo y complejizándolo; permitiéndoles así, tener experiencias en estas actividades, a la vez que proveen a los estudiantes los instrumentos de mediación adecuado.

En los ambientes interculturales y pluritecnológicos se debe reconocer y asumir que los pueblos indígenas son portadores milenarios de conocimientos y saberes, que sus culturas enriquecen la cultura global de Latinoamérica. Son de gran valía los valores éticos, humanos y espirituales que los pueblos indígenas trasmiten. La cultura y la relación que los pueblos indígenas asumen con el ambiente -su cosmovisión y cosmogonía- son fuentes inagotables y constituyen una energía revitalizadora y sustentables de saberes y de conocimientos que, pueden ser fuentes científicas en el ámbito teórico y práctico para el autodesarrollo en el marco de una nueva relación horizontal con el mundo desde su pluridiversidad. 


\section{Metodología}

El enfoque de esta investigación es cuantitativo ya que este paradigma ayudó al tratamiento de la información de los datos a través de la categorización y descripción de las propiedades, características y perfiles de las personas, grupos, comunidades, procesos y objetos o cualquier otro fenómeno que se someta a un análisis (Hernández, Fernández y Baptista, 2010). El diseño es de naturaleza descriptivo, porque recoge información de manera independiente o conjunta sobre los conceptos o las variables a las que se refieren, es decir, describen las tendencias de un grupo o población.

\subsection{Participantes}

La aplicación del cuestionario online se llevó a cabo con una muestra de 83 profesores de Educación Secundaria de Nicaragua. La edad media de los participantes fue de 33 años, con un rango comprendido entre los 29 y los 45 años. El 28\% de los profesores fueron mujeres y el $72 \%$ restante fueron hombres.

\subsection{Variables e instrumentos}

El método para recolectar la información es la encuesta a través de un cuestionario online con una escala de Likert que valora los aspectos: tecnológicos, cognitivos y aprendizaje, enseñanza, diversidad en la población, contenidos disciplinarios, campo del conocimiento y diversidad como orientación. Todo ello para identificar la percepción del profesorado de matemáticas respecto a los ambientes educativos de las matemáticas. Es importante mencionar que se utilizó la plataforma Google Formulario para aplicar dicho instrumento.

\subsection{Procedimiento}

La administración del instrumento se realizó por parte de los autores a inicio del segundo semestre académico, 2020. Tuvo un carácter anónimo y fueron complementados por los sujetos la muestra de forma online. Con anterioridad a la toma de los datos, se obtuvo tanto el consentimiento previo, libre e informado del profesorado.

\subsection{Análisis}

La reconstrucción y descripción del estudio se logró gracias a la integración de los datos obtenidos a través del instrumento Online incorporado en el marco metodológico. También se tomó en cuenta los niveles de mediación de las variables mediante un análisis descriptivo a través del análisis estadístico utilizando el software Statistical Package for the Social Sciences (SPSS). En relación con el análisis de figuras y cuadros, se tomaron los datos idénticos de los mismos niveles para que la información fuera veraz, objetiva y que los resultados de la investigación no tuviera sesgo alguno. 
Igualmente se realizó el análisis de la confiabilidad y validez de la escala de Likert por medio del alfa de Cronbach que, es un coeficiente que permite medir la fiabilidad de la escala con un resultado de 0.96 , lo cual determinó un alto grado de fiabilidad, por tanto, el instrumento que se aplicó al profesorado es confiable.

\section{Resultados y discusión}

El primer tema del cuestionario está relacionado con el aspecto tecnológico que se promueve en el ambiente educativo como proceso de la práctica docente. Por tales razones, se hacen preguntas respecto a sí en los ambientes aprendizaje se definen la relación: del uso de objetos virtuales de aprendizaje (OVA), el planteamiento teórico y práctico para el uso de objetos virtuales de aprendizaje y las condiciones necesarias para el uso de estos elementos tecnológicos en la práctica docente.

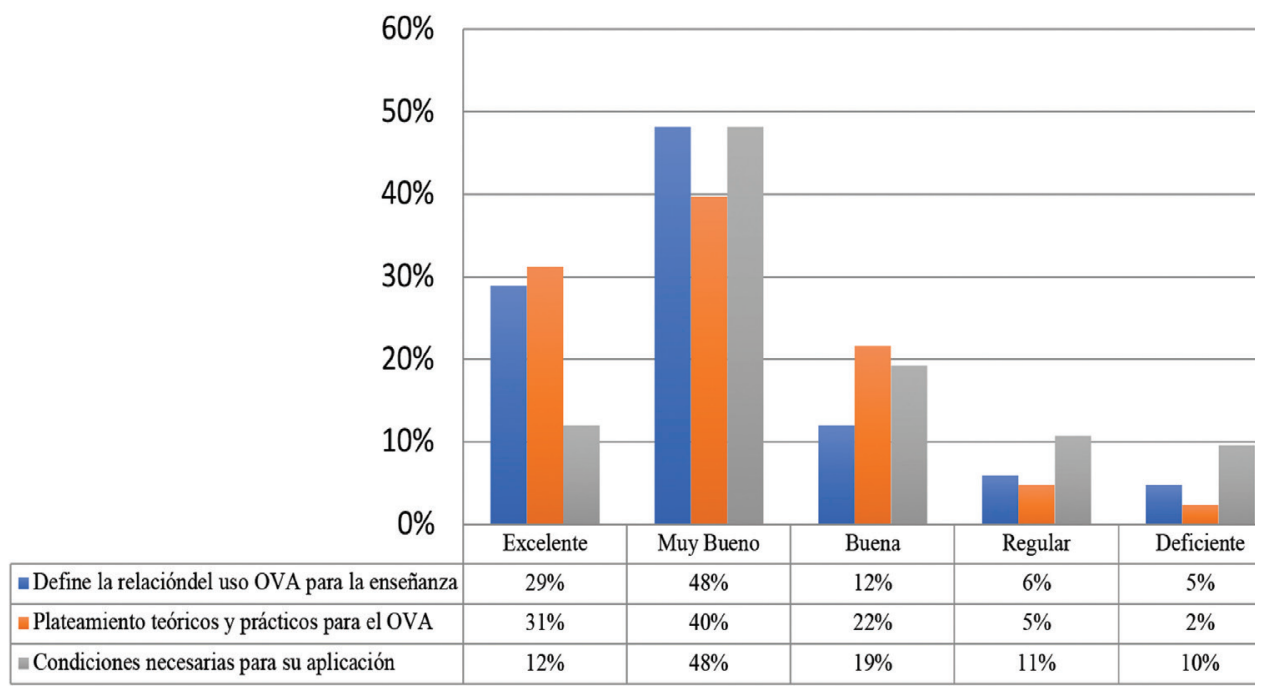

Figura no. 1. El ambiente de aprendizaje y su relación con los aspectos tecnológicos.

En la figura 1, se verifica que el $77 \%$ de los profesores valoran positivamente que en el ambiente educativo se defina el uso de los objetos virtuales de aprendizaje para la práctica docente en el aula. De los 83 profesores inquiridos, el $71 \%$ tiene una tendencia de apreciación entre "excelente y muy buena" en que, el ambiente de aprendizaje exista planteamientos teóricos y prácticos para la utilización de los objetos virtuales de aprendizaje. Se debe destacar que el $60 \%$ de los profesores consideran que en la institución académica existen las condiciones necesarias para la aplicación del ambiente de aprendizaje con aspectos tecnológicos, ya que hay ordenadores asignados a los profesores, acceso a internet, laboratorio de computación, una sala de medios con recursos digitales, plataforma virtual Moodle y sobre todo capacitación para el 


\section{EDUCACIÓN}

profesorado para promover el uso y construcción de materiales didácticos para la práctica docente.

El segundo tema del cuestionario fue la relación de los aspectos cognitivos y de aprendizaje con el ambiente de aprendizaje. Por tales razones, se consultó a los profesores sí en el ambiente de aprendizaje se define: un enfoque cognitivo, capacidades, habilidades y destrezas, acciones cognitivas de acuerdo con la diversidad, determinación del propósito de si misma, actividades cognitivas del sujeto, muestra funcionalidad y aplicabilidad en las actividades y explica la flexibilidad y adecuabilidad para la cognición.

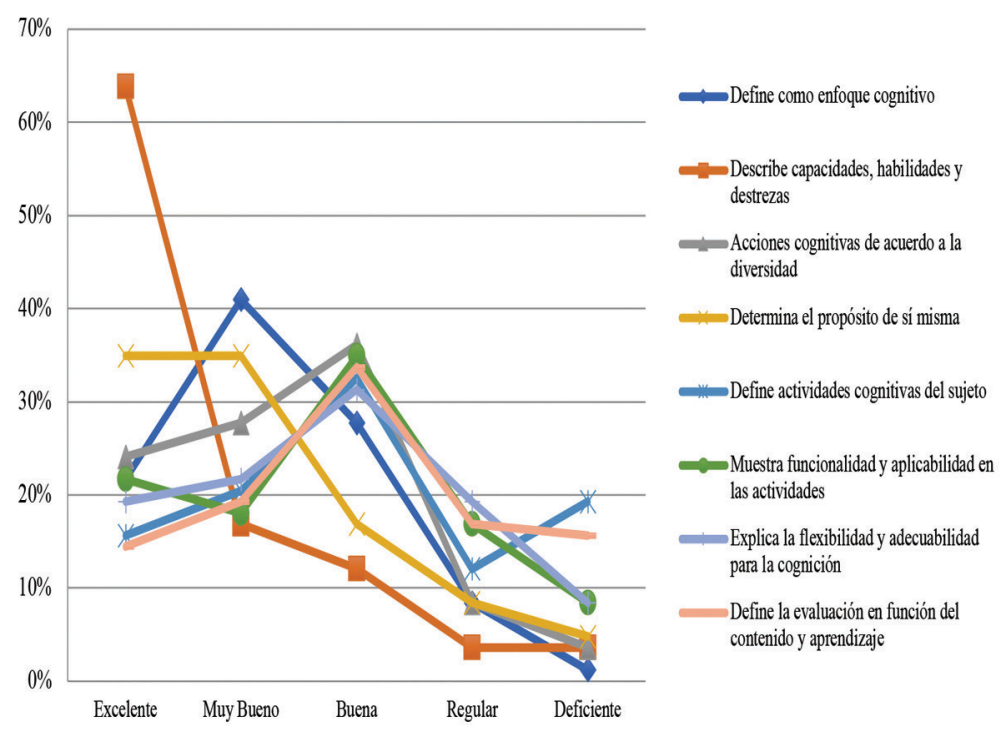

Figura no. 2. El ambiente de aprendizaje y su relación con los aspectos de cognición y aprendizaje

En la figura 2, mostramos una tendencia positiva hacia el ambiente de aprendizaje y su relación con los aspectos de cognición y aprendizaje. Observamos, que el 63\% de los profesores opinan, que en el ambiente se define un enfoque cognitivo entre excelente y muy bueno, lo que indica que la determinación del enfoque cognitivo ayudaría al desarrollo de la comprensión de los procesos matemáticos favoreciendo al aprendizaje de los estudiantes. Referente a que, si en el ambiente de aprendizaje se describen capacidades, habilidades y destreza, el $64 \%$ de los profesores valoran que si, porque hay una intención de aprendizaje de los contenidos, expresado en términos de objetivos, finalidades o propósitos de acuerdo con la visión pedagógica-didáctica. Sobre las acciones cognitivas de acuerdo a la diversidad en el aula, el 52\% de los profesores opinan la existencia del enfoque de atención a la diversidad en el ambiente de aprendizaje y que es bueno que se fomenten acciones como los grupos y diálogos interactivos en una práctica matemática. 
Relativo si en el ambiente de aprendizaje se determina el objetivo o propósito de si mismo, ante lo cual los profesores se perfilaron en una tendencia del $70 \%$ entre "excelente y muy bueno" esto debido a que los objetivos de aprendizaje se plantean en términos de la adquisición de instrumentos de la práctica de enseñar poniendo especial énfasis en los procesos y menos en los resultados. Sobre las definiciones de actividades cognitivas del sujeto al hacer uso de este en el ambiente de aprendizaje, el $69 \%$ de los profesores esta en una tendencia de "excelente y bueno" que se promuevan este tipo interacción ya es un aspecto importante reconocer que se aprende una interacción con uno mismo y con los otros, y que estos otros representan la diversidad como valor pedagógico y didáctico. También podemos observar que el $75 \%$ de los profesores creen que es "excelente y bueno", que un ambiente de aprendizaje sea un instrumento funcional, aplicable, flexible y adecuada para promover y satisfacer las necesidades cognitivas a las poblaciones a la cual se dirige. Finalmente, un $67 \%$ de los profesores opinan que el ambiente de aprendizaje define una propuesta de evaluación en función del contenido y tipo de aprendizaje.

El tercer tema del cuestionario fue la relación con los aspectos de enseñanza en el ambiente de aprendizaje, por consiguiente, se les preguntó a los profesores dos aspectos importantes sobre si en la unidad didáctica se establece el rol del profesor en el uso de las situaciones didácticas; y la relación de las guías de aprendizaje con el rol implementado en la unidad didáctica.

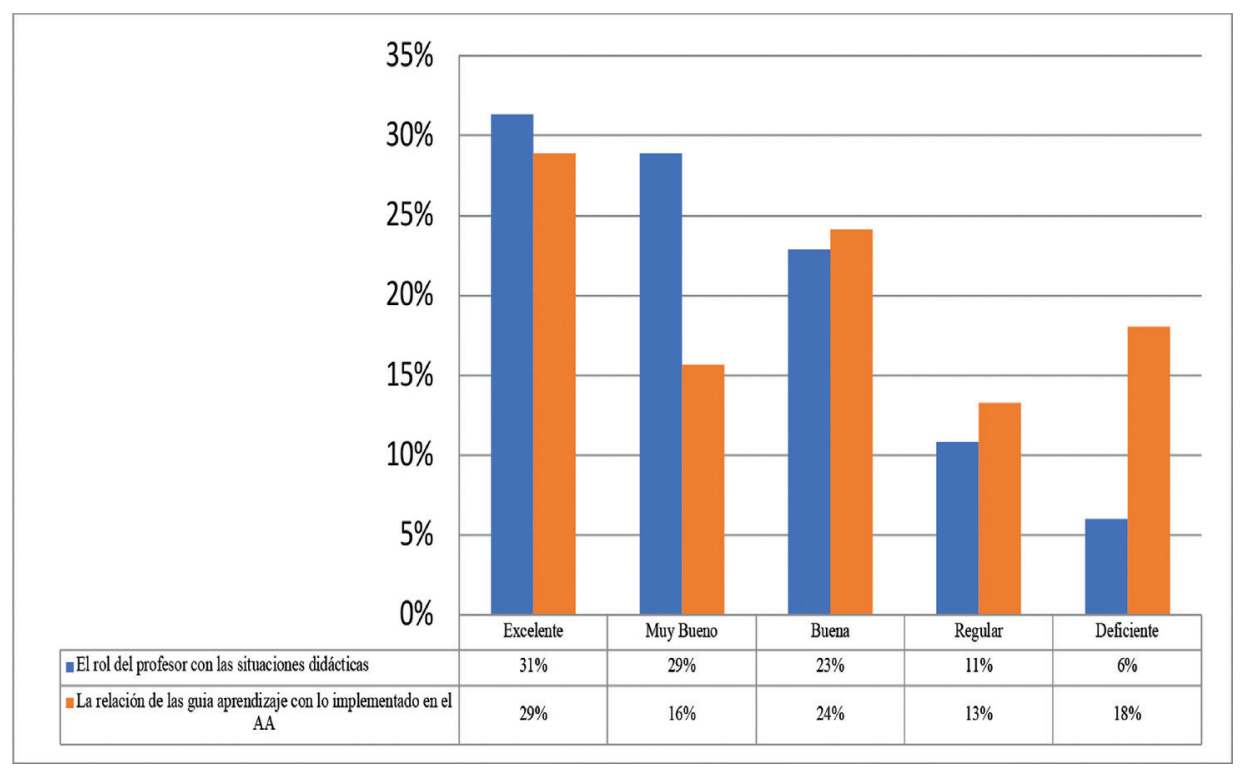

Figura no. 3. El ambiente de aprendizaje y la relación con los aspectos de enseñanza. 
La figura 3, muestra una inclinación entre "excelente y muy buena" ambas suman el 60\% de la valoración de los profesores indicando la pertinencia del rol del profesor con las situaciones didácticas, dicho rol corresponde a la puesta en marcha del diseño planeado, es decir, la gestión didáctica del profesor en términos de la ejecución de las actividades diseñadas en el ambiente de aprendizaje. Otro aspecto a destacar es la relación de las guías de aprendizaje con lo implementado en el ambiente de aprendizaje, en la figura visualizamos que el $69 \%$ de los profesores valoran positivamente que el rol del profesor en las guías de aprendizaje es del monitoreo de la ejecución de estas guías y, en general, verificar la adecuación de la disposición espacial y social en el aula para todos los estudiantes y observar el uso efectivo del recurso de mediación.

El cuarto tema del cuestionario es la relación del ambiente de aprendizaje con los aspectos de diversidad en la población. Por tanto, se consultó si en el ambiente de aprendizaje se definen: situaciones didácticas para poblaciones diversas; rasgos de diversidad a la población que se dirige; la accesibilidad para estudiantes indígenas, afrodescendientes y mestizo; y la flexibilidad para las poblaciones diversas.

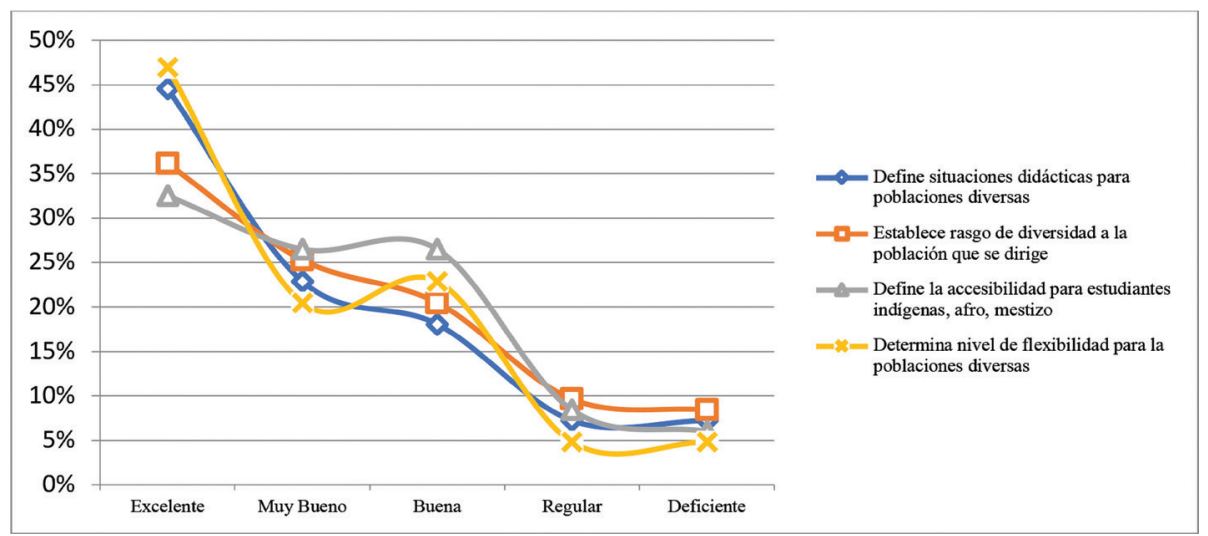

Figura no. 4. El ambiente de aprendizaje y la relación con los aspectos de diversidad en la población.

La figura 4, muestra apreciaciones positivas por parte de los profesores hacia el ambiente de aprendizaje en relación con aspectos de la diversidad en la población: el $67 \%$ de los profesores opina que se promueven interacciones de situaciones didácticas para poblaciones diversas; $61 \%$ de los profesores puntualiza en que el ambiente de aprendizaje se establece los rasgo de diversidad a la población que se dirige; el 59\% de los profesores expresa que el ambiente de aprendizaje define la accesibilidad para estudiantes indígenas, afrodescendientes y mestizo; y finalmente un $67 \%$ de los profesores visualiza que el ambiente de aprendizaje es un instrumento en que se determina el nivel de flexibilidad para la poblaciones diversas. La acción de construir un ambiente de aprendizaje con aspecto de diversidad es una relación con el diseño para todos. 
El quinto tema abordado en el cuestionario fue la relación del ambiente de aprendizaje con los contenidos disciplinarios, para ello se pregunto tres aspectos importantes: se determinan conceptos, temas y problemas significativos; asimismo, muestra la pertinencia de los contenidos disciplinarios y define los contenidos considerando edad, etnia, y género.

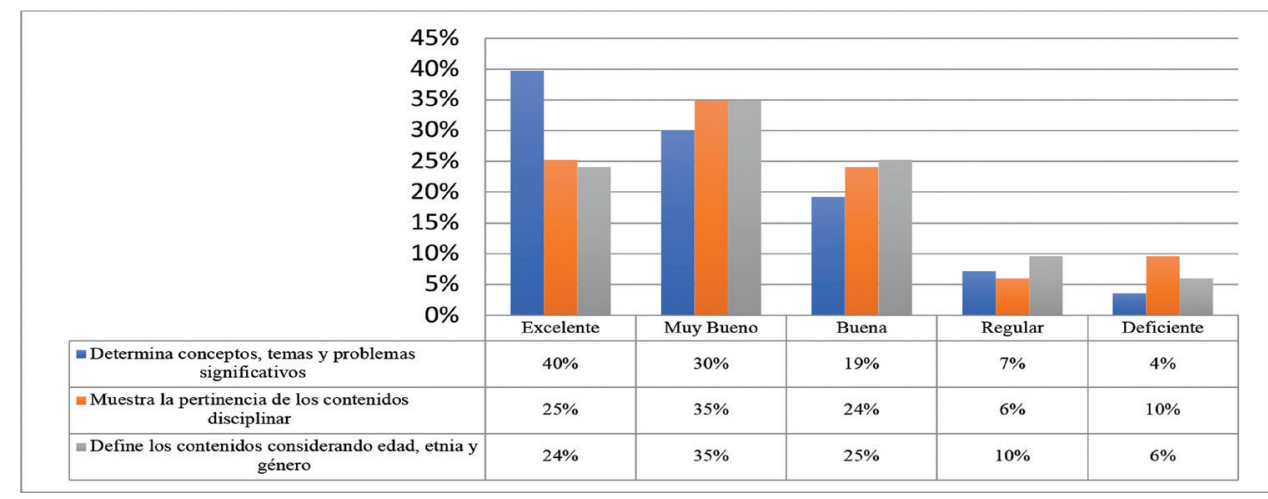

Figura no. 5. El ambiente de aprendizaje y la relación con los contenidos disciplinarios.

En la figura 5, podemos apreciar que los profesores perciben que el ambiente de aprendizaje tiene una estrecha relación con los contenidos disciplinarios está relación se refleja en una escala de "excelente y buena": el $89 \%$ de los profesores percibe que en el ambiente de aprendizaje se determina conceptos básicos, temas o tópicos centrales o los problemas significativos en el uso de ella misma, un $84 \%$ de los profesores distingue que en el ambiente de aprendizaje se muestra la pertinencia de los contenidos disciplinarios, también un $84 \%$ de los profesores cree que en el ambiente de aprendizaje se refleja el uso de los contenidos disciplinarios según el tipo de población considerando edad, género y etnia. El conjunto de contenidos disciplinarios definido en el ambiente de aprendizaje, cubre el abanico de las necesidades matemáticas de los estudiantes como base para su vida y para ampliar su horizonte de las matemáticas. También, los contenidos instaurados en el ambiente de aprendizaje pueden relacionarse con áreas como la aritmética, el álgebra, o la geometría y sus correspondientes sub-áreas que, reflejan las ramas definidas del pensamiento matemático y que facilitan el desarrollo de un programa de enseñanza estructurado. En general, los contenidos disciplinarios aseguran que las tareas utilizadas para la evaluación tienen una distribución suficiente a lo largo del ambiente de aprendizaje, pero al mismo tiempo en un número no muy amplio que evite una división excesiva.

El sexto tema en el cuestionario fue la relación del ambiente de aprendizaje con el campo del conocimiento, para ello se consultó sí en el ambiente educativo se definen los aspectos siguientes: 


\section{EDUCACIÓN}

Cuadro no.1: El ambiente de aprendizaje y la relación con el campo del conocimiento

\begin{tabular}{|c|c|c|c|c|c|}
\hline Campo del Conocimiento (Enfoque Didáctico) & Excelente & Muy Bueno & Buena & Regular & Deficiente \\
\hline Define el enfoque didáctico & $31 \%$ & $29 \%$ & $29 \%$ & $6 \%$ & $5 \%$ \\
\hline $\begin{array}{l}\text { Define múltiples experiencias -prácticas, } \\
\text { ficticias, hipotéticas }\end{array}$ & $18 \%$ & $27 \%$ & $22 \%$ & $20 \%$ & $13 \%$ \\
\hline Define múltiples sistemas de representación & $18 \%$ & $30 \%$ & $30 \%$ & $16 \%$ & $6 \%$ \\
\hline Define múltiples ambientes de aprendizaje & $17 \%$ & $30 \%$ & $20 \%$ & $18 \%$ & $14 \%$ \\
\hline $\begin{array}{l}\text { Define ambientes de aprendizaje para la } \\
\text { diversidad }\end{array}$ & $30 \%$ & $28 \%$ & $17 \%$ & $12 \%$ & $13 \%$ \\
\hline $\begin{array}{c}\text { Define la utilización de OVA con participa- } \\
\text { ción activa y propositiva }\end{array}$ & $33 \%$ & $16 \%$ & $22 \%$ & $13 \%$ & $17 \%$ \\
\hline $\begin{array}{l}\text { Define su propósito a la didáctica de la mate- } \\
\text { mática especializada }\end{array}$ & $27 \%$ & $34 \%$ & $30 \%$ & $8 \%$ & $1 \%$ \\
\hline $\begin{array}{l}\text { Las situaciones didácticas están de acorde a } \\
\text { lo planteamientos del AA }\end{array}$ & $29 \%$ & $22 \%$ & $34 \%$ & $10 \%$ & $6 \%$ \\
\hline Promedio en las puntuaciones & $25 \%$ & $27 \%$ & $25 \%$ & $13 \%$ & $9 \%$ \\
\hline
\end{tabular}

Podemos apreciar en el cuadro 1, que los profesores perciben entre "excelente y muy bueno" la relación del ambiente educativo con el campo del conocimiento, sobresaliendo aspectos como: define su propósito de manera acorde con la didáctica especializada (matemática) con un 61\%; el enfoque didáctico a ser utilizado en las situaciones didácticas con un 60\%; los ambientes de aprendizaje propiciados en el aula, deben distinguirse alentar la interacción entre personas con rasgos diversos: étnicos, físicos, cognitivos con un 58\%; y presentan actividades de enseñanza de acordes con los planteamientos teóricos-didácticos presentes en el ambiente de aprendizaje con un $51 \%$ del agrado de los profesores. Por otra parte, se puede apreciar en la escala de "excelente y muy buena" una tendencia entre $45 \%$ a $49 \%$ en aspectos como: el abordaje de los contenidos, se requiere de utilizar múltiples experiencias -prácticas, ficticias, hipotéticas; los contenidos deben de abordarse alentado el uso de múltiples formas de representación: graficas, orales, icónicas, diagramáticas; el abordaje de los contenidos, debe considerarse el uso de diferentes ambientes de aprendizajes: grupal; individual; presencial, virtual; estructurado, poco estructurado. La utilización de objetos virtuales de aprendizaje en el aula, debiera darse con la participación activa y propositiva de los estudiantes.

El séptimo tema en el cuestionario fue la relación del ambiente de aprendizaje con el aspecto de la diversidad como orientación, para ello se consultó aspectos de que si en el ambiente de aprendizaje se definen: una filosofía de atención a la diversidad y si los dispositivos tecnológicos definen los rasgos de diversidad atendido. 


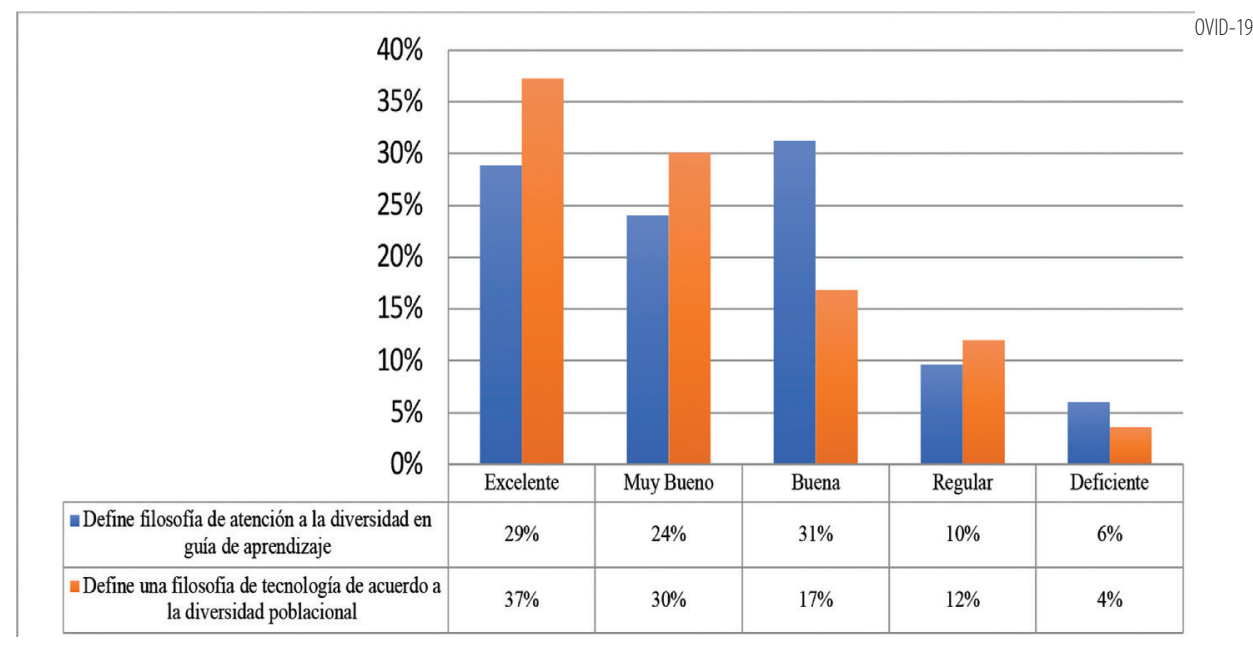

Figura no. 6. El ambiente de aprendizaje y la relación con los aspectos de diversidad como orientación.

La figura 6, muestra una tendencia positiva hacia que el ambiente de aprendizaje se promuevan los aspectos de diversidad como orientación, en una escala de excelente y muy bueno encontramos que el 53\% de los profesores hacen referencia que el ambiente de aprendizaje se define una filosofía de atención a la diversidad y que esta la sustenta en las guías de aprendizaje, así como un $67 \%$ de los profesores creen que en el ambiente de aprendizaje se determina con especificidad el uso de dispositivos electrónicos, de acuerdo con el rasgo de la diversidad atendida. Estos dos aspectos nos sitúan en las formas de concentración de la atención de poblaciones en contexto diversos, es decir, un diseño con todos, el cual inicia a partir del planteamiento de situaciones-problemas con todos, en el momento de vida apropiado, en el lugar necesario y con los recursos adecuados.

De un modo general, los profesores tienen una visión positiva del ambiente de aprendizaje situándolo como un recurso educativo para la formación de estudiante en contexto de diversidad. Claramente el diseño didáctico opera con la pareja diseño para todos, diseño con todos, para los estudiantes en cualquier condición sensorial, cultural, lingüística o socioeconómica, interactuando juntos aprenden matemáticas y para que los profesores tengan recursos educativos en un ambiente de coexistencia con la diversidad de poblaciones.

\section{Conclusiones}

Los resultados obtenidos en esta investigación deben ser entendidos en el contexto de la enseñanza de las matemáticas, que busca responder a la heterogeneidad de los participantes en un ambiente de aprendizaje, pero integrando algunos aspectos: tecnológicos, cognitivos, aprendizaje y enseñanza, diversidad en las poblaciones, contenidos disciplinarios, campo del conocimiento y diversidad como orientación. Además, es un ambiente de aprendizaje que se inserta en el ámbito de la enseñan de las matemáticas de forma virtual ante el COVID-19. 
La percepción general del profesorado en el ambiente del aprendizaje de las matemáticas en el mismo es muy positiva. Destaca la valoración sobre el aspecto tecnológico. La integración de las tecnologías en un ambiente de aprendizaje para la enseñanza de las matemáticas muestra que son herramientas importantes para fortalecer considerablemente la aprehensión de conocimiento y enriquecimiento cognitivo (Flores y Rojas, 2013), esto constituye en la percepción y el grado de planteamiento teórico para la utilización de las tecnologías en el ambiente de aprendizaje y por ende en el proceso de enseñanza y aprendizaje.

Otro aspecto importante es la percepción del profesorado frente a la cognición y aprendizaje que se instaura en el ambiente de aprendizaje, puesto que el ambiente constituye un sistema de prácticas compartidas en términos de comunidades de aprendizaje con énfasis al reconocimiento de la diversidad. En este sentido, los contenidos disciplinarios y enfoque didáctico (campo de conocimiento) responden a la diversidad como orientación, es decir, reconocen a una estructura simbólica que permite transformar las relaciones matemáticas presentadas y formular adecuadas respuestas a las preguntas derivadas.

Este estudio no busca únicamente presentar una radiografía del pensamiento del profesor en relación con los ambientes de aprendizaje de las matemáticas, sino también potencializar la construcción de diseños con todos y diseño para todos, considerando la idea de incluir a la diversidad y diseñar para ella hasta arribar en el diseño con la diversidad y desde ella.

En este sentido, los resultados de esta investigación avalan la utilización de ambientes aprendizaje para la enseñanza de las matemáticas con el reconocimiento de la diversidad en la didáctica de las matemáticas. Por lo tanto, los posibles mecanismos que se pueden considerar para afianzar el reconocimiento de la diversidad en el profesorado de matemáticas de forma que se incremente su grado de apropiación y se obtenga un impacto positivo son los siguientes:

1. Formación del profesorado: Formar el profesorado de matemática desde la perspectiva de comunidades de aprendizajes; creación y recreación de conocimientos, saberes y prácticas; estrategias de acompañamiento e incidencia social y comunitaria. Dado que a través de ellos se puede, por una parte, transferir y generar nuevo conocimiento y por otra, lograr mejores prácticas que permitan enlazar el conocimiento endógeno con el conocimiento exógeno.

2. Construcción de ambientes de aprendizaje: Es necesario la construcción de ambientes de aprendizajes sustentados en una comunidad de aprendizaje, partiendo de la idea que la comunidad de practica se constituye, sí y sólo sí entre sus miembros existe un compromiso mutuo de realizar una cierta empresa, lo que lleva a una práctica compartida en la que se genera aprendizaje compartido y significativo. 
3. Reconocimiento de la diversidad: El papel que juega la comunidad en la universidad, los sabios, sabias, ancianos, ancianas y autoridades tradicionales y no tradicionales en los procesos educativos; la búsqueda de nuevos paradigma en los enfoques pedagógicos y metodológicos para le generación de conocimientos para el Buen Vivir de los pueblos, la espiritualidad y el pensamiento mágico religioso; la interculturalidad, la educación intercultural y la educación multilingüe; la igualdad de género; el papel central de la investigación y la innovación; la articulación entre la teoría y la práctica; y una educación para todos y todas a lo largo de toda la vida.

4. Resiliencia afectiva e intercultural: Es necesario trabajar el conjunto de habilidades de procedencia psicológica que influyen positivamente en las actitudes, emociones y creencias, ya sea para afrontar una adversidad con el manejo de las emociones mediante el diálogo inter epistémico y complementariedad de conocimientos endógenos y exógenos.

\section{Lista de referencias}

Calderón, D., León, O., e INCI (2010). Incidencia de las representaciones sociales sobre la ceguera en el acceso del niño ciego a la educación básica primaria. Bogotá: INCI-UD.

Calderón, D., y León, O. (2010). Bilingualism of Colombian Deaf Children in the Teaching-Learning of Mathematics in the Year of Elementary School. Colombian Applied Linguistics Journal, 12(2), 9-24.

Cano, M., y Lledo, A. (1995). Espacio, comunicación y aprendizaje. Sevilla: Díada Editorial S. L.

Fiegehen, L. (2005). Repitencia y deserción universitaria en América Latina. En IESALC (Eds.). Seminario sobre rezago y la Deserción Universitaria en América Latina y el Caribe (156-168). CINDA-IELSALC.

Flores, W., O. (2018). Evaluación de los aprendizajes en la educación superior intercultural. Revista Electrónica De Conocimientos, Saberes Y Prácticas, 1(2), 54-65. https://doi.org/10.30698/recsp.v1i2.12

Flores, W., O., y López, E. (2016). Recursos didácticos y tecnológicos para la enseñanza de la integral definida en el modelo de Universidad Comunitaria Intercultural. Ciencia e Interculturalidad, 18(1), 63-78. https://doi.org/10.5377/ rci.v18i1.3050

Flores, W.O., y Rojas, N. (2013). Integración de las tecnologías de la información y comunicación en la enseñanza universitaria. Horizontes Pedagógicos, 15(1), 46-57. 


\section{EDUCACIÓN}

Hernández, R., Fernández, C., y Baptista, M. (2010). Metodología de la investigación. México: McGraw-Hill.

IESALC. (2008). Tendencias de la Educación Superior en América Latina y el Caribe. París: UNESCO.

Karsenti, T. (2013). The MOOC. What the research says. International Journal of Technologies in Higher Education, 10(2), 23-37.

León, O., Bonilla, M., Romero, J., Gil, D., Correal, M., Ávila, C., C., Bacca, J., Cavanzo, A., Guevara, C., Saiz, M., García, R., Saíz, E., Rojas, N., Peralta, M., Flores, W., O., y Márquez, H. (2014). Referentes Curriculares con Incorporación de Tecnologías para la Formación del Profesorado de Matemática en y para la Diversidad. Bogotá: Universidad Distrital Francisco José de Caldas. 